\title{
Photometric observations and frequency analysis of the $\delta$ Scuti NT Hya
}

\author{
D. Sinachopoulos and P. Gavras \\ Institute of Astronomy and Astrophysics, National Observatory of Athens \\ I. Metaxa and Bas. Pavlou, GR-15236 Athens, Greece
}

\begin{abstract}
NT Hya is a $\delta$ Scuti star discovered by Hipparcos. About 2200 observations were acquired with the 30 inch SAAO telescope in the nights of February 2,3 and 4, 2006 in $V$ filter. These data show four pulsation frequencies of the star. Two of them may form a doublet separated by $0.64 \mathrm{c} / \mathrm{d}(\Delta \mathrm{f} / \mathrm{f}=9 \%)$ with similar amplitudes. The period listed in the Hipparcos Catalogue is between the doublet frequencies.
\end{abstract}

\section{Introduction and Observations}

NT Hya (HD 78422) is listed in the revised catalogue of $\delta$ Sct stars by Rodriguez et al. (2000) as a F0 variable star with mean $V=7.37$, amplitude 0.06 magnitudes and frequency $6.940 \mathrm{c} / \mathrm{d}$. Its entry was based on information taken from the catalogue presented by Kazarovets et al. (1999). Their name-list introduces GCVS names for 3153 variable stars discovered by the Hipparcos mission (HIP 44813). The mentioned information of the Rodriguez et al. catalogue was adopted from the corresponding Hipparcos catalogue entry (ESA 1997). We tried to determine its high frequency pulsations by means of photometric observations. These were scheduled in the week 31/1/2006 - 6/2/2006 with the 30 inch telescope of the South African Astronomical Observatory, using the UCT photometer and $\mathrm{V}$ filter.

Due to weather conditions observations were made only during a large part of the third and the fifth nights and the photometric fourth night of this period. During these nights 2214 measurements of NT Hya were collected. The exposure time was always 10 seconds. Table 1 gives a journal of observations providing an overview of date, number of data points and photometric hours per 


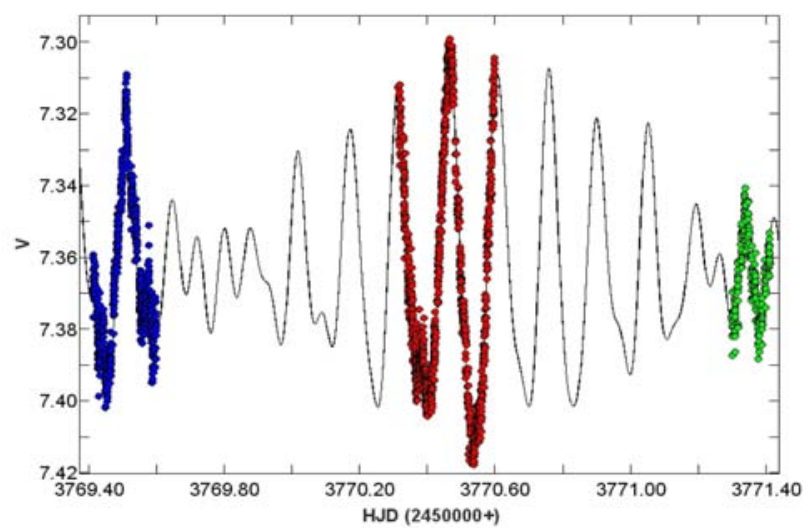

Figure 1: 2006 observations of NT Hya, together with the fit of the frequencies found

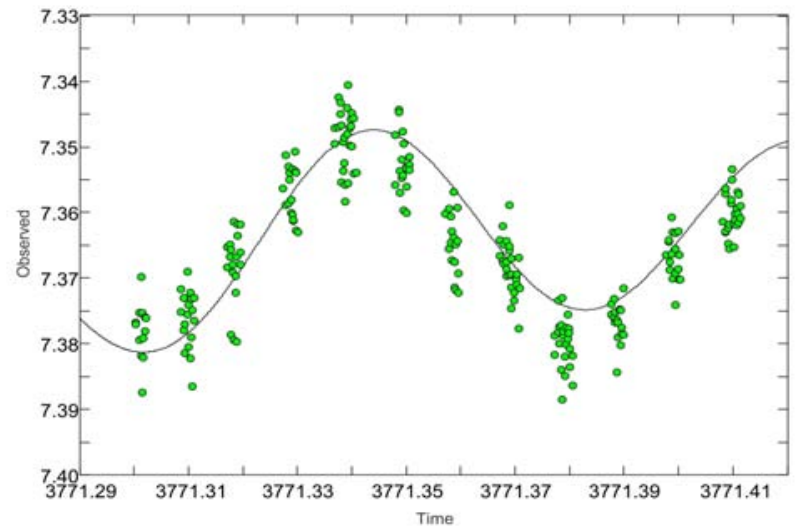

Figure 2: The observations of the 3rd night and the fit of the four frequencies

\begin{tabular}{rrrrr}
\multicolumn{5}{c}{ Table 1: Journal of Observations } \\
\hline HJD & Photometric & \multicolumn{2}{c}{ Data Points } \\
$2450000+$ & Hours & NT Hya & Comp. & Sky \\
\hline 3769 & 4.77 & 724 & 290 & 54 \\
3770 & 7.42 & 1209 & 581 & 81 \\
3771 & 3.25 & 281 & 155 & 46 \\
\hline
\end{tabular}


Table 2: Observed frequencies for NT Hya (V-data)

\begin{tabular}{rrrr}
\hline Id. & $\begin{array}{r}\text { Frequency } \\
{[\mathrm{c} / \mathrm{d}]}\end{array}$ & $\begin{array}{r}\text { Semi-ampl. } \\
{[\mathrm{mag}]}\end{array}$ & $\begin{array}{r}\text { Phase } \\
\text { [cycles] }\end{array}$ \\
\hline$f_{1}$ & 6.50 & 0.024 & 0.893 \\
$f_{2}$ & 7.14 & 0.023 & 0.666 \\
$f_{3}$ & 13.59 & 0.009 & 0.067 \\
$f_{4}$ & 10.43 & 0.006 & 0.129 \\
\hline
\end{tabular}

night. The comparison star was the HIP $44940 \mathrm{~V}=7.8$ and $(\mathrm{B}-\mathrm{V})=-0.08$ and an anonymous $\mathrm{V}=8.5$ star was used as a check star. The standard observing sequence proposed by Henden \& Kaitchuck (1982) for monitoring stars varying rapidly was followed. The comparison star was observed every 15 minutes and 7-10 exposures have been obtained each time. The sky was observed at the beginning and the end of the observations, as well as after each observation of the comparison. The check star was mainly observed during the third clear night and its limited number of observations show random deviations around 0.005 mag relative to the comparison star.

\section{Frequency analysis}

At a first glance the light curve shows intense variations from night to night. The largest peak-to-peak amplitude is $0.12 \mathrm{mag}$. Figure 1 shows the complete data set of our 2006 observations of NT Hya.

The Fourier analysis was performed using Period04 (Lenz \& Breger 2005). Four frequencies were detected and listed in Table 2 together with their semiamplitudes, in order of decreasing amplitude.

\section{Discussion and Conclusion}

We note that the difference of $0.64 \mathrm{c} / \mathrm{d}$ between the two frequencies, $f_{1}$ and $f_{2}$, is below the proposed frequency resolution of $1.5 / \mathrm{dT}$ limit by Loumos \& Deeming (1978) and therefore need to be confirmed. $f_{3}$ could be a combination frequency of $f_{1}$ and $f_{2}$. Adding $f_{4}$ to the other three fitted frequencies reduces the residuals between the fit and the observations to $0.0058 \mathrm{mag}$, although minor deviations between the fit and the data remain for the third night, as shown in Fig. 2. 
The main period of NT Hya given by the Hipparcos catalogue is between the two frequencies $f_{1}$ and $f_{2}$. The ratio of these frequencies indicates non-radial pulsation for at least one of the modes $\left(f_{1} / f_{2}=0.91\right)$.

Follow-up observations are needed for giving an clear answer to the nature and the relations of $f_{1}, f_{2}$ and $f_{3}$.

Acknowledgments. Authors are extremely thankful for anonymous referees remarks in general and for those on the section of frequency analysis in particular.

\section{References}

ESA 1997, The Hipparcos and Tycho Catalogues, ESA SP-1200

Henden, A. A., \& Kaitchuck, R. H. 1982, Astronomical Photometry, Van Nostrand Reinhold Publ., New York

Lenz, P., \& Breger, M. 2005, CoAst 146, 53

Loumos, G. L., \& Deeming, T. J. 1978, ApSS 56, 285

Rodriguez, E., Lopez-Gonzalez, M. J., \& Lopez de Coca, P. 2000, A\&AS 144, 469

Kazarovets, A. V., Samus, N. N., Durlevich, O. V., et al. 1999, IBVS 4659, 1 